\section{Photorefractive Polymer Device Records High-Resolution 3D Holographic Images}

Holograms hold a special place in the popular imagination, from the cover of National Geographic to episodes of Star Trek. But three-dimensional holography is fast becoming a reality for high-value applications, including biomedical imaging of living tissue. Devices for such 3D holographic imaging have so far been limited by either slow response times or low sensitivity. Now, in an article in the August 1 issue of Optics Letters (p. 1914), a group from the University of Manchester in the United Kingdom has introduced holographic imaging with a photorefractive polymer device that is both fast and has high 3D resolution, even through a scattering medium similar to organic tissue.

A hologram is formed by creating ("writing") a pattern of refractive-index contrast inside a material. When the material is later illuminated with a "read" laser, this pattern diffracts the coherent laser light and creates the hologram. A common technique for recording refractiveindex patterns is to use a photorefractive material in which incident light from the object being holographed liberates charge carriers in the material, which get trapped at points of low- or high-incident intensity. The electric field of these trapped charge carriers then modifies the local index of refraction through the electro-optic effect.

The Manchester group-P. Dean, M.R. Dickinson, and D.P. West-used as their holographic medium a photorefractive polymer host poly( $N$-vinylcarbazole) and doped it with $50 \mathrm{wt} \%$ of the electrooptic chromophore 1-(2'-ethylhexyloxy)2,5-dimethyl-4-(4" -nitrophenylazo) benzene and $2 \mathrm{wt} \%$ of the sensitizer 2,4 , 7-trinitro-9-fluorenone dimalenitrile to enhance the photorefractive effect. The holographic reference beam intensity was $161 \mathrm{~mW} / \mathrm{cm}^{2}$, while the object beam intensity was $0.7 \mathrm{~mW} / \mathrm{cm}^{2}$, and the reading beam intensity was $0.5 \mathrm{~mW} / \mathrm{cm}^{2}$. With an exposure time of only a few seconds, they were able to record a high-resolution hologram of an object with transverse details as small as $42 \mu \mathrm{m}$ and a depth resolution of $15 \mu \mathrm{m}$ over an object area of $0.42 \mathrm{~mm}^{2}$. Then, to demonstrate the usefulness of this technique for biomedical imaging, the researchers placed a suspension of polystyrene microspheres between the holographed object and the recording laser, which simulated the optical scattering effects of an intervening layer of biological tissue. The optical depth of the microspheres corresponded to seven scattering mean free paths (round-trip). With this layer present, the required recording time increased to $20 \mathrm{~s}$, but the hologram resolution remained very high.

What makes the group's technique particularly noteworthy is that it operates at a near-infrared wavelength of $794 \mathrm{~nm}$, which is only weakly absorbed by biological tissue. This, combined with the technique's relatively fast time scale and high spatial resolution at low optical intensity, make it an especially promising advance toward the clinical goal of holographic biomedical imaging.

\section{COLIN MCCORMICK}

\section{Self-Assembled InAs/InP Quantum Dots Transform into Quantum Rings without Capping}

Quantum rings-ring-shaped solid pipes with nanometer diameters-have recently been under intense investigation. In some quantum dot (QD) fabrication efforts, capping self-organized InAs QDs by a thin GaAs layer followed by a growth interruption results in a change of shape from a dot to a ring. Two competing models, thermodynamic and kinetic, have been proposed to explain the growth phenomena of these quantum rings. In the August 10 issue of NanoLetters (p. 1541; DOI: $10.1021 /$ nl050646v), J. Sormunen and colleagues from Helsinki University of Technology, Finland, have reported their fabrication of InAs/InP rings. They have debated that a third mechanism, a Group V exchange reaction, is responsible for such growth.

The samples in their experiment were prepared by metalorganic vapor-phase epitaxy (MOVPE) under atmospheric pressure. Hydrogen was used as a carrier gas, with trimethylindium (TMI), ter-

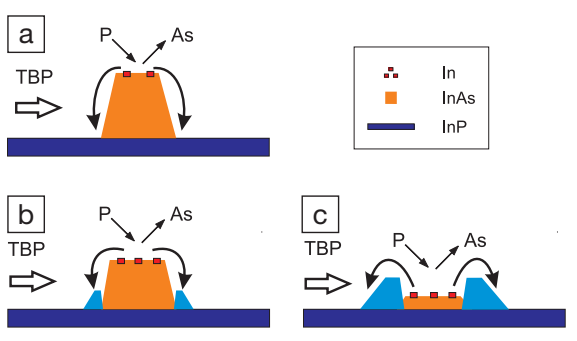

Figure 1. Schematic diagram of the dotto-ring transformation of an InAs/InP quantum dot. (a) In atoms are released by As/P exchange and migrate outward from the InAs island. (b) In atoms are reincorporated at the interface of the quantum dot and the flat surface. (c) Material redistribution continues until the initial InAs island has disappeared. TBP is tertiarybutylphosphine. Reprinted with permission from NanoLetters 5(8)(2005) p. 1541; DOl: 10.1021/nl050646v. (C) 2005 American Chemical Society. tiarybutylphosphine (TBP), and tertiarybutylarsine (TBA) as precursors. The growth process is explained in Figure 1. The substrate was first annealed at $650^{\circ} \mathrm{C}$, then a $100 \mathrm{~nm}$ InP buffer layer was grown at $640^{\circ} \mathrm{C}$. Next, the temperature was reduced to $560^{\circ} \mathrm{C}$ and $0.6-1.0$ monolayers of InAs was deposited, followed by the self-organized growth of an InAs quantum dot. Following the formation of the InAs QDs, a flush was performed in TBA, after which the Group V precursor was switched to TBP. Figure 1 shows the proposed quantum-ring formation process. When TBA is switched to TBP, As / $P$ exchange releases In atoms from the InAs lattice (Figure 1a). The In adatoms migrate toward the base of the InAs island, where the lateral lattice constant is closer to that of InP (Figure 1b), and are reincorporated at an energetically more favorable site. Material redistribution continues (Figure 1c) until the initial InAs island has vanished.

The researchers conclude that because the quantum rings were fabricated uncapped, As/P exchange plays an important role, in contrast to the thermodynamic and kinetic models. Because of Group V exchange reactions, the uncapped rings were assumed to consist of $\operatorname{InAs} \mathrm{P}_{y} \mathrm{P}_{1-y}$.

VIVEK RANJAN

\section{Gradient Chemical Micropatterning Enables Fabrication of Reference Substrates for Calibration of Scanning Surface Nanometrology}

Scanning probe microscopy (SPM) techniques (e.g., atomic force microscopy, friction force microscopy, chemical force microscopy, and atomic force acoustic microscopy) are important tools for surface nanometrology. However, their use as tools for examining surface chemistry, particularly for materials processing and technologies including nanolithography masks, micro- and nanoelectromechanical systems, microfluidics, and tissue scaffolds, requires detailed knowledge of surface energy variations across the nanostructures. Accurate measurement of surface energy is critically affected by the condition of probe tips and their possible chemical contamination, which cannot always be easily determined. Consequently, the accuracy of such scanning probe measurements would be greatly facilitated by the existence of a reference substrate with surface energy gradients spanning the values of interest that could be used as a calibration tool.

Most current methods to impart substrates with tailored surface energy and chemical patterns involve only "single 